\title{
Proton NMR Relaxation Study of Thermotropic Copolyesters
}

\author{
Kazuo SATo, Shun-ichi SuzUKI, Nobuyuki InABA, \\ and Hideya MURAI \\ Polymer Research Laboratory, Idemitsu Petrochemical Co., Ltd., \\ Anesakikaigan, Ichihara, Chiba 299-01, Japan
}

(Received September 9, 1986)

\begin{abstract}
Hydroxybenzoic acid/poly(ethylene terephtharate) (PHB/PET) and $p$-hydroxybenzoic acid/6-hydroxy-2-naphthoic acid (PHB/PHN) thermotropic copolyesters were investigated in the temperature range from -120 to $180^{\circ} \mathrm{C}$ by observing the proton magnetic relaxation times $T_{1}, T_{1 \rho}$, and the line width. In the temperature dependence of $T_{1 \rho}$ of PHB/PET two minima of the relaxation times were observed. The one at higher temperature corresponds to the glass transition, and the other at lower temperature $\left(40^{\circ} \mathrm{C}\right)$ is considered to result from the phenylene group motion. For PHB/PHN, the transition similar to the low temperature one of $\mathrm{PHB} / \mathrm{PET}$ was observed. The transition temperature of $\mathrm{PHB} / \mathrm{PHN}$ is higher by $30-40^{\circ} \mathrm{C}$ as compared with PHB/PET due to the rigid structure. From analysis of line width, it was found that there are two motional regions for both copolyesters, and the activation energies could be obtained. The $T_{1}$ minimum associated with the glass transition was observed in the $T_{1}$ experiment. The mesophase formation seems to narrow the distribution of the correlation times of motion.

KEY WORDS Liquid Crystalline Polymer / Molecular. Motion $/{ }^{1} \mathrm{H}$ Nuclear Magnetic Relaxation / Thermotropic Copolyester /
\end{abstract}

Recently, much attention has been focused upon thermotropic copolyesters which provide a potential for developing high modulus materials. Since the pioneering work of Jackson and Kuhfuss ${ }^{1}$ was reported, there have been many activities in the synthesis of thermotropic main chain copolyesters. Physical properties of their copolyesters have been investigated in most cases by thermal analysis and morphological observations. ${ }^{2}$ To understand the high dynamic modulus resulting from self-reinforcing property of such thermotropic copolyesters, it is essential to investigate the effect of liquid crystal formation on molecular motions. The molecular motion and relaxation have been studied by dielectric dispersion, ${ }^{3}$ ESR spin probe, ${ }^{4}$ and broad line $\mathrm{NMR}^{5}$ etc. However, information is limited, and further study is needed. We investigated thermotropic copolyesters by observing ${ }^{1} \mathrm{H}$ NMR relaxation times, $T_{1}, T_{1}$, and $T_{2}$ in the temperature range from -120 to $180^{\circ} \mathrm{C}$. Copolyesters studied were $p$-hydroxybenzoic acid/poly(ethylene terephtharate) (PHB/PET), and $p$-hydroxybenzoic acid/6-hydroxy-2naphthoic acid (PHB/PHN) copolyesters. The mole fractions of PHB in PHB/PET copolyester were 30,60 , and $80 \%$. PHB/PHN copolyester contains $73 \mathrm{~mol} \%$ PHB. These copolyesters are, hereafter, denoted by PHB/PET30, PHB/ PET60, PHB/PET80, and PHB/PHN, respectively. These copolyesters except PHB/PET30 are known to show nematic liquid crystalline behavior. We reported the quite high orientational order of the mesogen in the fiber and film of PHB/PET60 by observing the proton dipolar splitting. ${ }^{6}$ In addition to knowledge about the molecular orientation, information concerning the molecular motion is expected to give insight into the nature of liquid crystalline copolyesters. 


\section{EXPERIMENTAL}

\section{Materials}

PHB/PET copolyesters were copolymers based on poly(ethylene terephtharate) modified with $p$-hydroxybenzoic acid of 30, 60, and $80 \mathrm{~mol}^{\%}$. PHB/PHN copolyester was a commercial product.

\section{NMR Measurement}

The instrument used was Bruker CXP-90 operating at $90 \mathrm{MHz}$ and equipped with a temperature variable unit B-VT1000. Temperature of the sample was varied from -120 to $180^{\circ} \mathrm{C}$. Pellets of a sample were packed into NMR tube, degassed under vacuum and then sealed off. Spin-spin relaxation was estimated as the line width at half height of the absorption spectrum obtained by Fourier transformation of FID. Spin-lattice relaxation times $T_{1}$ and $T_{1 \rho}$ in static and rotating frames were measured by the usual inversion recovery $\left(180^{\circ}-\tau-90^{\circ}\right.$ pulses $)$ and $90^{\circ}(x)-90^{\circ}$ phase shifted locking pulse methods, respectively. The locking field in $T_{1 \rho}$ experiment was 10 Gauss.

\section{RESULTS AND DISCUSSION}

\section{PHB/PET Copolyester}

Typical spectra of PHB/PET60 are shown in Figure 1. At higher temperature, similar spectra to those reported by McFarlane et al. ${ }^{7}$ were observed. The dipolar splitting is due to the liquid crystalline nature of this copolyester, and the spectra correspond to powder spectra. The central narrow line appearing above about $120^{\circ} \mathrm{C}$ is attributed to the isotropic mobile protons, the fraction of which increases with rising temperature. This result is in agreement with that obtained by McFarlane et al.

Temperature dependence of the line width at half height of PHB/PET60 is shown in Figure 2. At higher temperatures where a narrow line appears, the line width of the broad component is taken as the line width of the spec-

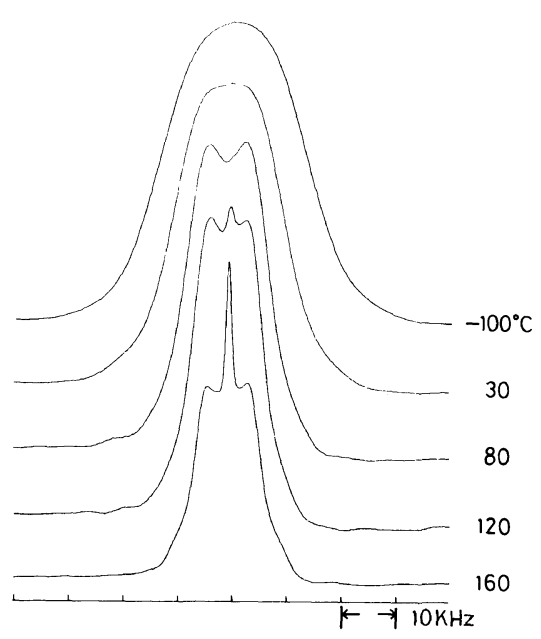

Figure 1. ${ }^{1} \mathrm{H}$ NMR spectra of PHB/PET60 copolyester measured at indicated temperatures.

trum. The line width is about $29 \mathrm{KHz}$ at low temperature, and gradually decreases with increasing temperature. The line width decreases rapidly at the temperatures between -40 and $80^{\circ} \mathrm{C}$, and then decreases gradually above $80^{\circ} \mathrm{C}$. The effect of motion on the line width can be analysed using the following phenomenological equation for motional narrowing in solids, ${ }^{8}$

$$
\ln (1 / W-1 / A)=-E_{\mathrm{a}} / k T+\ln (1 / B-1 / A)(1)
$$

where, $W$ is the line width at temperature $T, E_{\mathrm{a}}$ is the motional activation energy, and $A$ and $B$ are the limiting values of the line width at low and high temperatures, respectively. The advantage in using eq 1 is that the temperature dependence of the line width can be analysed even when the value of $B$ cannot be easily defined and that the activation energy is estimated without being influenced by the distribution of correlation times of motion. Figure 3 shows experimental line widths of PHB/PET60 together with PHB/PET30 plotted as a function of the inverse of temperature according to eq 1. Two distinct motional regions are observed above and below $70^{\circ} \mathrm{C}$. This temperature corresponds to the glass transition temperature of PHB/PET60 determined by 


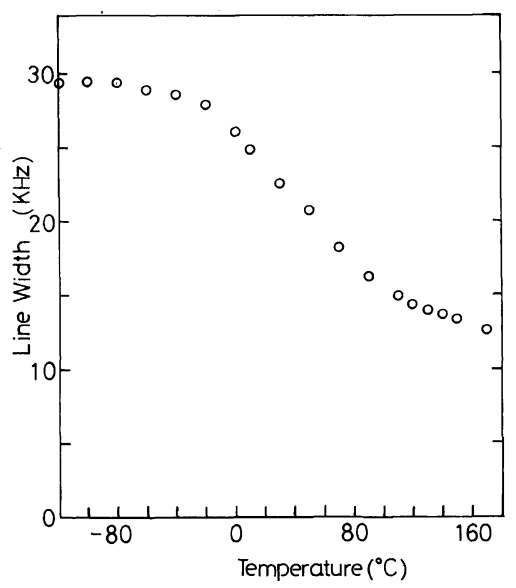

Figure 2. Line width at half height of the broad component of PHB/PET60 copolyester as a function of temperature.

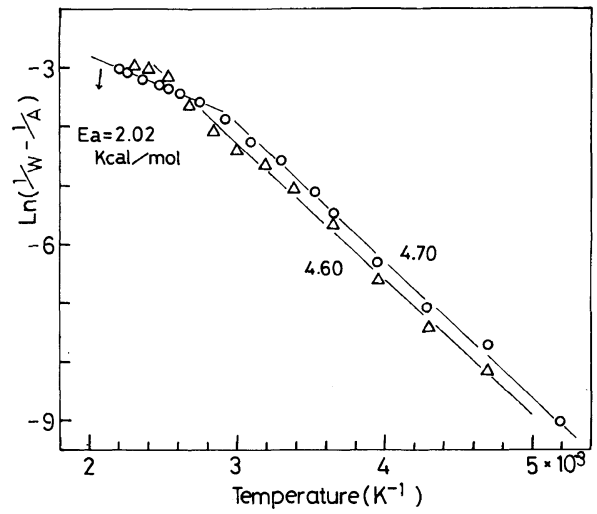

Figure 3. Plot of line width as a function of the inverse of temperature according to eq 1 . The line width at $-120^{\circ} \mathrm{C}$ was taken as $A$ in eq 1 . $\bigcirc$, PHB/PET60; $\triangle$, PHB/PET30.

dynamic mechanical measurements. The motional process which causes the narrowing of the line width is considered to be different above and below $T_{\mathrm{g}}$. The activation energies in the low and high temperature regions are 4.70 and $2.02 \mathrm{kcal} \mathrm{mol}^{-1}$, respectively. In the case of PHB/PET30, two motional regions are also observed; however, the high temperature region is obscure probably due to higher glass transition temperature, $90^{\circ} \mathrm{C}$, determined by dynamic mechanical measurements. The acti-

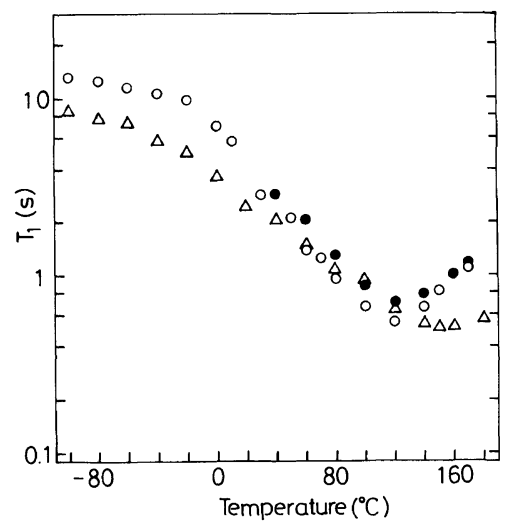

Figure 4. Plot of spin-lattice relaxation time $T_{1}$ of PHB/PET copolyesters as a function of temperature. PHB/PET80; O, PHB/PET60; $\triangle$, PHB/PET30.

vation energy $4.60 \mathrm{kcal} \mathrm{mol}^{-1}$ in the low temperature region agrees with that of $\mathrm{PHB} /$ PET60, indicating the motional process not to depend upon whether a liquid crystalline mesophase exists or not.

Temperature dependences of spin-lattice relaxation times of PHB/PET30, 60, and 80 are shown in Figure 4. In the whole temperature range studied, the magnetization recovery was exponential and single $T_{1}$ value was obtained. This may result from the effective spin diffusion. $T_{1}$ minima observed at $120^{\circ} \mathrm{C}$ for $\mathrm{PHB}$ PET60 and 80 and $150^{\circ} \mathrm{C}$ for PHB/PET30 correspond to the glass transition of respective copolyesters. It is of interest to note that $T_{1}$ minimum of PHB/PET30 is broad compared with those of PHB/PET60 and 80. It is reasonable to consider that this phenomena is attributed to the broad distribution of correlation times of PHB/PET30. The ordered mesophase formation in PHB/PET60 and 80 may result in the narrow distribution of correlation times and the sharp minimum of $T_{1}$.

We would like to discuss the results of the $T_{1 \rho}$ experiment in the following. Figure 5 shows the temperature dependence of $T_{1 \rho}$ of PHB/PET copolyesters. At low temperatures below $-30^{\circ} \mathrm{C}$, two $T_{1 \rho}$ 's were observed, indicating the spin diffusion does not act effec- 


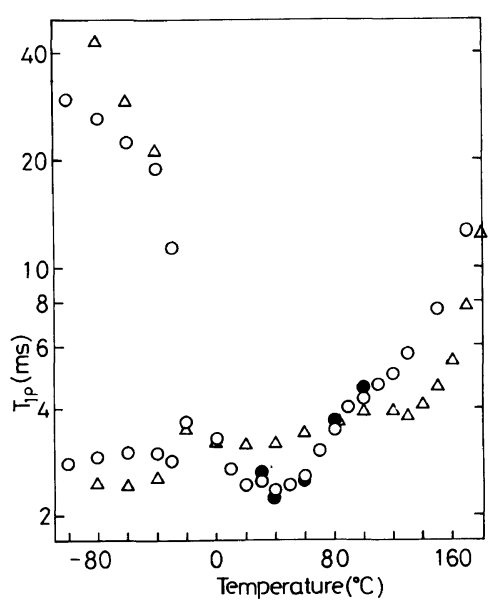

Figure 5. Plot of spin-lattice relaxation time $T_{1 \rho}$ in the rotating frame $\left(H_{1}=10\right.$ Gauss $)$ of PHB/PET copolyesters as a function of temperature. - PHB/PET80; $\bigcirc$, PHB/PET60; $\triangle$, PHB/PET30.

tively in $T_{1 \rho}$ time scale. The fraction of component with long $T_{1 \rho}$ increases with increasing PHB content. Therefore, it is reasonable to attribute the long $T_{1 \rho}$ component to the rigid aromatic constituent. The long $T_{1 \rho}$ decreases with rising temperature and above $-20^{\circ} \mathrm{C}$ the magnetization decay in the rotating frame becomes exponential and single $T_{1 \rho}$ was obtained. The $T_{1 \rho}$ minimum observed at $120^{\circ} \mathrm{C}$ for PHB/PET30 and the $T_{1 \rho}$ shoulder at $90^{\circ} \mathrm{C}$ for $\mathrm{PHB} / \mathrm{PET} 60$ correspond to the respective glass transition temperatures of these copolyesters. The $T_{1 \rho}$ minimum indicating a molecular relaxational motion was observed at $40^{\circ} \mathrm{C}$, which is considered to be the low temperature motion observed in the line width study. It is interesting to note that this relaxation was not detected by the $T_{1}$ experiment. In the $T_{1}$ experiment, molecular motion with a correlation frequency of $10^{7}$ $10^{8} \mathrm{~Hz}$ influences effectively nuclear relaxation; on the other hand, in the $T_{1 \rho}$ experiment, motion with correlation frequency $10^{4}-10^{5}$ $\mathrm{Hz}$ is much effective. ${ }^{9}$ Therefore, we consider the relaxation at $40^{\circ} \mathrm{C}$ to be due to the relatively slow motion.

One of the possible motional mode may be the phenyl ring $\pi$-flip motion about $\mathrm{C}_{1}-\mathrm{C}_{4}$ axis. Recently, such a motion has been proposed for polymers containing aromatic groups in the main chain such as polycarbonate, ${ }^{10}$ poly(butylene terephtharate) ${ }^{11}$ and poly(arylene ether sulfones) ${ }^{12}$ on the basis of solid state ${ }^{2} \mathrm{H}$ and ${ }^{13} \mathrm{C}$ NMR studies. It has been found that the phenyl ring flip motions are relatively slow. Jelinski et al. ${ }^{11}$ estimated the activation energy of the $\pi$-flip process of poly(butylene terephtharate) to be 5.9 $\mathrm{kcal} \mathrm{mol}^{-1}$, comparable to the values obtained in this experiment. The $T_{1 \rho}$ minimum of $\mathrm{PHB} /$ PET60 is much narrower than that of PHB/ PET30, also indicating the narrow distribution of correlation times ${ }^{13}$ in the former. In the ordered state, the mode of motion may be considered to become more simple.

\section{PHB/PHN Copolyester}

Figure 6 shows the relationship between the temperature and $T_{1 \rho}$ of $\mathrm{PHB} / \mathrm{PHN}$ copolyester. The temperature dependence is similar to those of PHB/PET copolyesters, but the temperature of $T_{1 \rho}$ minimum is higher by 30 $40^{\circ} \mathrm{C}$ and the values of long $T_{1 \rho}$ at low temperature are larger by about one order. This is attributed to the rigid structure of $\mathrm{PHB} / \mathrm{PHN}$. The similar temperature dependence suggests that the relaxation observed in the experiment originates from the molecular motion of the common mesogenic unit rather than different spacer units. The $\pi$-flip motion of the phenyl ring may also be the possible motional process in $\mathrm{PHB} / \mathrm{PHN}$ as in PHB/PET.

The line width of $\mathrm{PHB} / \mathrm{PHN}$ plotted as a function of temperature according to eq 1 is shown in Figure 7. Two motional regions were also observed above and below $60^{\circ} \mathrm{C}$, but this temperature does not correspond to the glass transition of the copolyester, but rather to the temperature of the $T_{1 \rho}$ minimum. Activation energies were estimated to be 6.74 and 3.08 $\mathrm{kcal} \mathrm{mol}^{-1}$ in low and high temperature regions, respectively. These values are larger than the corresponding values of PHB/PET, 


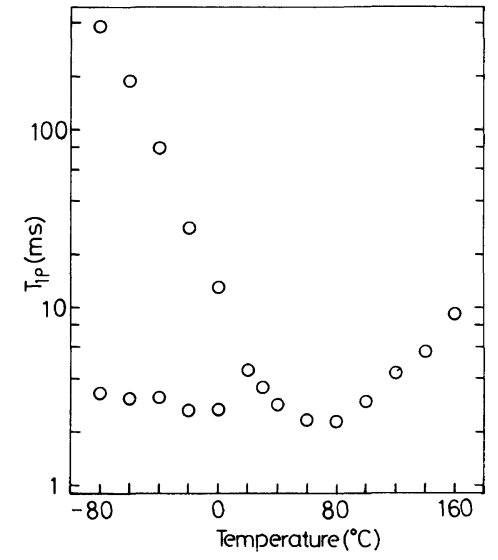

Figure 6. Plot of spin-lattice relaxation time $T_{1 \rho}$ in the rotating frame ( $H_{1}=10$ Gauss) of $\mathrm{PHB} / \mathrm{PHN}$ copolyester as a function of temperature.

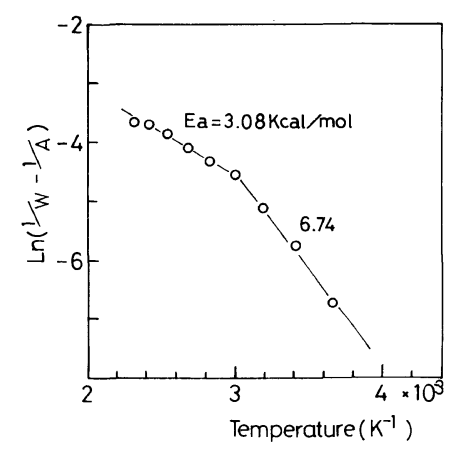

Figure 7. Plot of line width of PHB/PHN copolyester as a function of the inverse of temperature according to eq 1 . The line width at $120^{\circ} \mathrm{C}$ was taken as $A$ in eq 1 .

indicating a rigid structure of $\mathrm{PHB} / \mathrm{PHN}$. The high temperature region associated with the high temperature side of the $T_{1 \rho}$ minimum has the lower activation energy. At present detailed motional mode is not clear. Motion of terminal groups may be one of the possible modes as suggested by Mitchell and Ishii. ${ }^{5}$ For PHB/PET the high temperature region above $T_{\mathrm{g}}$ is probably due to the quasi liquid like motion of the ethylene group.

\section{CONCLUSIONS}

We investigated two thermotropic copolyes- ters, PHB/PET (PHB: 30, 60, and $80 \mathrm{~mol}^{\circ} \%$ ), and PHB/PHN (PHB: $73 \mathrm{~mol}_{\%}^{\circ}$ ) by observing proton magnetic relaxation times, $T_{1}, T_{1 \rho}$, and line width in the wide temperature range. By analysis of the line width on the basis of Hendrickson-Bray equation, ${ }^{8}$ it was concluded that the two motional regions exist for both copolyesters. The activation energies in the high and low temperature motional regions of $\mathrm{PHB} / \mathrm{PHN}$ are larger than those of the corresponding values of PHB/PET, due to rigid molecular structure of the former copolyester. In the $T_{1 \rho}$ experiment of PHB/PET copolyesters, two minima indicating the molecular relaxational motions were observed. These two relaxational mode correspond to the high and low temperature motional regions observed in the line width study. The relaxation at high temperature corresponds to the glass transition, and the other at $40^{\circ} \mathrm{C}$ is considered to be ascribed to the $\pi$-flip motion of phenylene group. The $T_{1}$ minimum associated with this motional relaxation was not observed, probably because the frequency of the motion is not so high $\left(10^{7}-10^{8} \mathrm{~Hz}\right)$ as to dominate the spin lattice relaxation in the static field. The $T_{1 \rho}$ minimum ascribed to the $\pi$-flip motion was also observed for PHB/ PHN, however, the temperature at the minimum becomes higher by $30-40^{\circ} \mathrm{C}$ compared with PHB/PET. The $T_{1}$ and $T_{1 \rho}$ minima of PHB60 and 80 were sharper than those of PHB/PET30 which does not exhibit the liquid crystalline nature. The sharp minimum of relaxation means the narrow distribution of correlation times of motion, indicating the ordered liquid crystal formation resulting in the simple motional mode.

\section{REFERENCES}

1. W. J. Jackson, Jr. and H. F. Kuhfuss, J. Polym. Sci., Polym. Chem. Ed., 14, 2043 (1976).

2. A. Cifferi, W. R. Krigbaum, and R. B. Meyers (Ed.), "Polymer Liquid Crystals," Academic Press, New York, N. Y., 1982.

3. G. S. Attard, G. Williams, G. W. Gray, D. Lacey, 
and P. A. Gemmel, Polymer, 27, 185 (1986); F. Laupretre, C. Noel, W. N. Jenkins, and G. Williams, Faraday Discuss. Chem. Soc., 79, 191 (1985).

4. P. Meurisse, C. Friedrich, M. Dvolaitzky, F. Laupretre, C. Noel, and L. Monnerie, Macromolecules, 17, 72 (1984).

5. G. R. Mitchell and F. Ishii, Polym. Commun., 26, 34 (1985).

6. K. Sato, N. Inaba, H. Murai, and S. Suzuki, submitted to Polym. J.

7. F. E. McFarlane, V. A. Nicely, and T. G. Davis, "Contemporary Topics in Polymer Science," Vol. 12, Plenum, New York, N. Y., 1977, p 109.

8. J. R. Hendrickson and P. J. Bray, J. Magn. Reson., 9,
341 (1973).

9. V. J. McBrierty and D. C. Douglass, J. Polym. Sci., Macromol. Rev., 16, 295 (1981).

10. J. Schaefer, E. O. Stejskal, R. A. Mckay, and W. T. Dixon, Macromolecules, 17, 1479 (1984); J. F. O. Gara, A. A. Jones, C. C. Hung, and P. T. Inglefield, Macromolecules, 18, 1117 (1985).

11. A. L. Cholli, J. J. Dumais, A. K. Engel, and L. W. Jelinski, Macromolecules, 17, 2399 (1984).

12. J. J. Dumais, A. L. Cholli, L. W. Jelinski, J. L. Hedrick, and J. E. McGrath, Macromolecules, 19, 1884 (1986).

13. T. M. Connor, Trans. Faraday Soc., 60, 1574 (1964). 